\title{
Impact of pulmonary exacerbations and lung function on generic health-related quality of life in patients with cystic fibrosis
}

\author{
Caitlyn T. Solem ${ }^{1 *}$, Montserrat Vera-Llonch², Sizhu Liu', Marc Botteman ${ }^{1}$ and Brenda Castiglione ${ }^{2}$
}

\begin{abstract}
Background: The analysis aimed to examine the impact of pulmonary exacerbations (PEs) and lung function on generic measures of HRQL in patients with cystic fibrosis (CF) using trial-based data.

Methods: In a 48-week randomized, placebo-controlled study of ivacaftor in patients $\geq 12$ years with CF and a G551D-CFTR mutation the relationship between PEs, PE-related hospitalizations and percent predicted forced expiratory volume in one second (ppFEV ${ }_{1}$ ) with EQ-5D measures (index and visual analog scale [VAS]) was examined in post-hoc analyses. Multivariate mixed-effects models were employed to describe the association of PEs, PE-related hospitalizations, and $\mathrm{ppFEV}$, on EQ-5D measures.

Results: One hundred sixty one patients (age: mean 25.5 [SD 9.5] years; baseline ppFEV 1 : 63.6 [16.4]) contributed 1,214 observations (ppFEV 1 : no lung dysfunction $[n=157]$, mild $[n=419]$, moderate $[n=572]$, severe $[n=66]$ ). Problems were most frequently reported on pain/discomfort, anxiety/depression, and usual activities EQ-5D items. The mean (SE) EQ-5D index nominally decreased (worsened) with worsening severity of lung dysfunction $(P=0.070)$ : 0.931 (0.023); mild: 0.923 (0.021); moderate: 0.904 (0.018); severe: 0.870 (0.020). 146 PEs were experienced by 72 patients, including 52 PEs (35.6 \%) that required hospitalization. Mean EQ-5D index and VAS scores were lowest (worst) within 1 week (before or after PE start) for PEs requiring hospitalization. Pulmonary exacerbations, PE-related hospitalizations, and ppFEV ${ }_{1}$ were significant predictors of EQ-5D index and VAS.
\end{abstract}

Conclusions: In a clinical study of patients with CF ( $\geq 12$ years of age and a G551D-CFTR mutation), PEs, primarily those requiring hospitalization, were associated with low EQ-5D index and VAS scores. The impact of ppFEV 1 was relatively smaller. Reducing PEs, in particular those requiring hospitalization, would likely improve HRQL among these patients.

Trial registration: ClinicalTrials.gov, NCT00909532; URL: clinicaltrials.gov, May 26, 2009

Keywords: Cystic fibrosis, EQ-5D, Lung function, Pulmonary exacerbation

\section{Background}

Health-related quality of life (HRQL) is an increasingly important multi-dimensional clinical outcome assessment which provides insights into the patient's experience of disease burden and the effects of medical interventions. Measures of HRQL are patient-reported and can be generic or disease-specific [1]. Disease-specific measures may be more sensitive to some of the symptoms experienced by patients but generic measures permit uniform

\footnotetext{
* Correspondence: csolem@pharmerit.com

${ }^{1}$ Pharmerit International, 4350 East West Hwy, Suite 430, Bethesda, MD 20814, USA

Full list of author information is available at the end of the article
}

comparisons across medical conditions and as such are necessary to aid decision making in evaluating the value of new treatments. As the landscape of therapies for cystic fibrosis (CF) expands, it is important to characterize the relationship between clinical and physiologic measures of disease and generic measures of HRQL.

Cystic fibrosis is an inherited, rare autosomal recessive disease that results in chronically debilitating morbidities and high premature mortality [2]. CF disease affects multiple organs in the body including the lung, pancreas, intestinal and biliary tracts, sweat glands and the reproductive system [3]. Patients with CF typically experience progressive loss of lung function ultimately resulting in 
respiratory failure and death [2]. A key characteristic affecting CF disease trajectory is the occurrence of pulmonary exacerbations (PEs), which require acute medical care and often hospitalization. Avoiding PEs is a foremost goal of CF treatment since exacerbation frequency is associated with lung function decline, greater likelihood of subsequent exacerbations and increased mortality [4]. Pulmonary exacerbations, particularly those that are severe, have been reported to impact HRQL [5-8]. Lung function as measured by forced expiratory volume $\left(\mathrm{FEV}_{1}\right)$ is an outcome measure in clinical studies of CF therapies and has been shown to be related to patient's survival and HRQL in cross-sectional and longitudinal studies $[8,9]$.

Research to date has reported mixed degrees of associations between several CF physiologic and clinical measures such as $\mathrm{FEV}_{1}$ and PEs, and generic and disease-specific HRQL measures (e.g. Cystic Fibrosis Questionnaire-Revised [CFQ-R] scores) [5-8] of CF symptoms and HRQL. Previous studies of patients with CF have reported utilities derived using time tradeoff [7, 10-12] and standard gamble [7, 13, 14] direct elicitation methods, and generic indirect measures including the health utilities index (HUI2 and HUI3) [7, 14, 15], and the EuroQol EQ-5D $[6,16,17]$ with varying results. Within CF there has been evidence of potential ceiling effects of generic measures such as the EQ-5D and insensitivity particularly to $\mathrm{FEV}_{1}$. While some of these studies have independently assessed the impact of lung function and PEs on generic measures of HRQL in CF, no studies to date have analyzed both of these key disease elements within the same model. In order to better assess this, the aim of this analysis is to examine the impact of PEs and lung function on generic HRQL as assessed by the EQ-5D-3 L questionnaire in patients aged 12 years and older with CF and a G551D mutation on at least 1 CFTR allele using data from the STRIVE clinical trial [18].

\section{Methods}

\section{Data source}

This analysis used data from a 48-week, Phase 3, international, multicenter, randomized, double-blind, placebocontrolled study (STRIVE) which was designed to evaluate the efficacy and safety of ivacaftor in patients aged 12 years and older with CF who had a G551D mutation on at least 1 CFTR allele [18]. Patients were included if they had an $\mathrm{FEV}_{1}$ of 40 to $90 \%$ (inclusive) of the predicted normal value for age-, gender-, and height-matched persons at screening $\left(\mathrm{ppFEV}_{1}\right)$. As reported in Ramsey et al. [18], "subjects were excluded if they had other illnesses that confounded the study results; ongoing illness; a pulmonary exacerbation or changes in therapy (including antibiotics) for pulmonary disease within 4 weeks before first dose of study drug; abnormal liver function tests, defined as 3 or more LFT parameters $>3$ times the upper limit of normal; or abnormal renal function tests. Subjects were also excluded if they had a history of prolonged QT/ QTc interval; history of solid organ or hematological transplantation; colonization with organisms associated with a more rapid decline in pulmonary status (e.g., $B$. cenocepacia, B. dolosa, and M. abcessus); concomitant use of any inhibitors or inducers of CYP3A4; or use of inhaled hypertonic saline treatment. Subjects were required to stop inhaled hypertonic saline treatment for at least 4 weeks prior to Day 1 (first dose of study drug)". Study assessments were conducted at baseline, day 15 , week 8 , and every 8 weeks thereafter through 48 weeks.

\section{Study measures}

\section{Health-related quality of life}

The EQ-5D-3 L [19] is a generic measure of HRQL that includes two components: a descriptive profile and a visual analogue scale (VAS). The descriptive profile includes five single-item dimensions (mobility, self-care, usual activities, pain/discomfort, and anxiety/depression), each with 3 levels of response (no problems, some problems, and extreme problems) that can be combined into a single score (index) which summarizes health status (i.e., utility) and is anchored at 0 (=death) and 1 (=perfect health). The index is calculated by an algorithm using patients' responses to the EQ-5D descriptive profile and preference weights for different health states ascertained from the general population of a country (herein, using values for the United Kingdom [UK]) [20].

The EQ-5D VAS records the respondent's self-rated health on a $20 \mathrm{~cm}$ vertical VAS with endpoints labeled "the best health you can imagine" and "the worst health you can imagine." [19]. This information can be used as a quantitative measure of health status as rated by the individual respondents.

\section{Lung function}

In the STRIVE study, $\mathrm{ppFEV}_{1}$ was the primary outcome measure. In our analyses, we characterize lung dysfunction respectively as: (1) no lung dysfunction (ppFEV $1 \geq 90 \%$ ), mild $\left(70 \leq \operatorname{ppFEV}_{1}<90 \%\right)$, moderate $\left(40 \leq \operatorname{ppFEV}_{1}<70 \%\right)$, and severe $\left(\mathrm{FEV}_{1}<40 \%\right)$; (2) deciles of $\mathrm{ppFEV}_{1}$; and (3) $\mathrm{ppFEV}_{1}$ treated as a continuous variable in multivariate analyses. Note that while patients were required to have at least mild lung dysfunction at entry to the trial, it was possible for patients to improve and have observations with no lung dysfunction at later points within the trial.

\section{Pulmonary exacerbations}

In the clinical study, PEs were defined as a change in antibiotic therapy for $\geq 4$ of 12 signs or pre-defined sino- 
pulmonary symptoms (change in sputum; new or increased hemoptysis; increased cough; increased dyspnea; malaise, fatigue, or lethargy; temperature above $38{ }^{\circ} \mathrm{C}$; anorexia or weight loss; sinus pain or tenderness; change in sinus discharge; change in physical examination of the chest; decrease in pulmonary function by $10 \%$; radiographic changes indicative of pulmonary infection). PE start and end dates and information on whether PEs required hospitalization or not was also collected.

\section{Statistical analysis}

EQ-5D measures were exploratory endpoints in the STRIVE clinical trial and their analyses were not prespecified therefore no statistical correction for multiplicity was undertaken. Accordingly, three sets of post-hoc analyses were subsequently conducted using trial-based data as described below. Analyses were undertaken using SAS/ STAT $^{\varpi}$ software, Versions 9.3 and 9.4 of the SAS System for Windows (Cary, NC, USA).

1) Relationship between EQ-5D measures and ppFEV $V_{1}$ Mean values and $95 \%$ confidence intervals for EQ-5D index and VAS scores were calculated using all observations across all study assessments and treatments and stratified by deciles of ppFEV 1 , and categories of lung dysfunction as described previously The proportions of patients with maximum value of EQ-5D index $(=1)$ and VAS score $(=100)$ respectively were calculated to assess ceiling effects.

2) EQ-5D measures and ppFEV $V_{1}$ among patients who experienced PEs

Data from patients who experienced at least one PE were included in this analysis. The periods prior and subsequent to the study defined PE start date were arbitrarily specified and grouped into pre-PE periods ( $>8$ weeks, $>4-8$ weeks and $>1-4$ weeks) and post-PE periods ( $>8$ weeks, $>4-8$ weeks, $>1-4$ weeks) respectively. The one-week pre- and post-PE start date constituted the reference category (i.e., "PE start period"). Mean (SD) duration of PEs was also calculated based on study defined PE start and end dates, overall and for patients for whom PEs required hospitalization and for those for whom they did not respectively (unadjusted).

Observations were visually graphed and overlaid with local regressions (LOESS) to depict the EQ-5D index and VAS scores over time respectively. Mixed-effects models for repeated measures (MMRM) were employed to generate (least squares [LS]) mean values for each period controlling for baseline $\mathrm{pFEV}_{1}$, age, sex, baseline body mass index (BMI), baseline sweat chloride, history of pancreatic insufficiency and baseline use of cycling antibiotics, and repeated observations. Models were also used to evaluate differences in EQ-5D index, VAS scores, and $\mathrm{ppFEV}_{1}$ between pre- and post-PE start periods and the reference period. A subgroup analysis was also conducted for patients for whom the absolute $\operatorname{ppFEV}_{1}$ value did not decline by 10 or more percentage points (from randomization to the closest $\mathrm{ppFEV}_{1}$ assessment) prior to the PE start date.

3) Association of PEs, ppFEV $V_{1}$ and EQ-5D measures Data from all patients (those who experienced PEs during the clinical study and those who did not) were included in this analysis. For patients who experienced one or more PEs, EQ-5D index, VAS scores and $\mathrm{ppFEV}_{1}$ between PE start and end dates (inclusive of those dates) were considered related to a PE. Observations that did not occur during a PE window were not considered PE-related. Multivariate (MMRM) analyses were undertaken to examine the association of experiencing a PE (requiring and not requiring hospitalization), and $\mathrm{ppFEV}_{1}$ (with linear and quadratic terms) with the EQ-5D measures as dependent variables. As a sensitivity analysis, regression models were also developed using US, Europe, Netherlands, and Belgium EQ-5D index value sets (i.e., preference weights), [21-23] as well as for VAS scores that were transformed into a health-state utility value using a previously reported equation [24].

\section{Ethics}

The STRIVE clinical trial ("A Phase 3, Randomized, Double-Blind, Placebo-Controlled, Parallel-Group Study to Evaluate the Efficacy and Safety of VX-770 in Subjects with Cystic Fibrosis and the G551D Mutation") protocol was reviewed and approved by the institutional review board at each participating center, and each subject provided written informed consent or written or oral assent.

\section{Results}

A total of 161 patients (age: mean [SD] 25.5 [9.5] years; baseline $\mathrm{ppFEV}_{1}$ : mean [SD] 63.6 [16.4]) contributed 1,214 sets of observations (EQ-5D measures and $\mathrm{ppFEV}_{1}$ ) over 48 weeks. Of the 1,214 $\mathrm{ppFEV}_{1}$ assessments (no lung dysfunction $[n=157]$, mild [ $n=149]$, moderate $[n=572]$, and severe $[n=66])$, all but 12 occurred on the same day as the EQ-5D measurements. Over the course of the study, patients most frequently reported problems with pain/ discomfort (20.2\%), followed by anxiety/ depression (16.4\%), and usual activities (14.1\%). The EQ-5D index was at its ceiling $(=1)$ for $67.5 \%$ (no lung dysfunction: $80.9 \%$; mild: $73.3 \%$; moderate: $62.1 \%$; severe: $45.5 \%$ ) of observations whereas the EQ-5D VAS was at the ceiling (VAS $=100$ ) for $5.6 \%$ (no lung dysfunction: $15.9 \%$; mild: $6.9 \%$; moderate: $2.4 \%$; severe: $0.0 \%$ ) of observations.

A total of 146 PEs were experienced by 72 (44.7\% of total 161) patients, including 52 PEs (35.6 \%) that 
required hospitalization. Mean (SD) duration was 30.0 (22.2) days for PEs requiring hospitalization and 20.6 (11.6) days for those not requiring hospitalization (11 PEs from 9 patients had missing PE end dates and were excluded). The baseline characteristics of patients who experienced a PE during the study and those who did not are summarized in Table 1. Patients who did not experience a PE during the study had on average a higher $\mathrm{ppFEV}_{1}$ at study initiation than those who experienced one or more PEs. Patients who experienced a PE requiring hospitalization were younger than those with a $\mathrm{PE}$ not requiring hospitalization and the mean $\operatorname{ppFEV}_{1}$ at study initiation did not differ between these two groups.

\section{EQ-5D measures and ppFEV}

Figure 1 shows EQ-5D index and VAS scores by ppFEV 1 decile. VAS scores appeared to be more discriminating of CF lung disease severity than the EQ-5D index. Within MMRM models, the EQ-5D index (mean, [SE]) nominally decreased (worsened) with increasing severity of lung dysfunction $(P=0.070)$ : no lung dysfunction: $0.931(0.023)$; mild: 0.923 (0.021); moderate: 0.904 (0.018); severe: 0.870 (0.020). Mean (SE) VAS scores followed a similar trend and were significantly different $(P<0.001)$ across $\mathrm{ppFEV}_{1}$ categories: no lung dysfunction: 85.2 (2.0); mild: 82.3 (1.8); moderate: 76.8 (1.6); severe: 73.3 (1.8).

\section{EQ-5D measures and ppFEV 1 by time since PE start}

EQ-5D index and VAS scores over time in relation to PE start are shown in Figs. 2 and 3 respectively. Nominal trends for declining (worsening) values in the pre- PE start periods can be inferred from visual inspection of the data (both index and VAS scores) suggesting detrimental changes in health status and symptoms in advance of the study defined PE start date, followed by a trend for recovery.
For PEs that required hospitalization mean EQ-5D index, VAS scores and $\mathrm{ppFEV}_{1}$ values were lowest (worst) during the PE start period within MMRM (Table 2); mean values for the EQ-5D and $\mathrm{ppFEV}_{1}$ measures are suggestive of a trend towards recovery following the PE start period although findings did not always reach statistical significance (compared to the PE start period). A similar pattern is suggested by findings for PEs that did not require hospitalization, but overall, results did not achieve statistical significance. Findings from a subanalysis which removed patients who had a $\operatorname{ppFEV}_{1}$ decline of 10 or more percentage points at the observation nearest to the PE start date were consistent with similar detrimental impacts on the EQ-5D index (for PEs that required hospitalization), suggesting that PEs affect HRQL beyond the $\operatorname{ppFEV}_{1}$ decline alone (data not shown).

In examining Fig. 3, one might question the "peak" in VAS scores for patients who experienced a PE requiring hospitalization immediately prior to the PE start date. Of the 6 observations that occurred within the 3 days prior to exacerbation, 3 had VAS values that were $>90$. Two of these three assessments were provided by patients with $\mathrm{ppFEV}_{1}<70 \%$ at the time of that visit; two additional observations, from different patients, also had a concurrent EQ-5D index $<1$.

Mean $\operatorname{ppFEV}_{1}$ was significantly lower during the PE start period as compared to the preceding and subsequent periods in particular for PEs that required hospitalization. Findings were similar for PEs that did not require hospitalization but did not always reach statistical significance when compared to the PE start period.

Between PE start and end dates, patients were most likely to report problems for usual activities ( $53.6 \%$ of observations), pain/discomfort (39.3\%) and mobility (32.1\%) for PEs that required hospitalization. For PEs not requiring hospitalization, the trend was similar but fewer patients reported problems: $35.7 \%$ for usual activities,

Table 1 Patient Characteristics at Baseline, by Pulmonary Exacerbations During Study

\begin{tabular}{|c|c|c|c|c|}
\hline & $\begin{array}{l}\text { No PE } \\
(n=89)\end{array}$ & $\begin{array}{l}\text { Any PE } \\
(n=72)\end{array}$ & $\begin{array}{l}\text { PE (No Hospitalization) } \\
(n=38)\end{array}$ & $\begin{array}{l}\text { PE (Hospitalization) } \\
(n=34)\end{array}$ \\
\hline Age, Mean (SD) & $25.5(10.2)$ & $25.4(8.8)$ & $28.3(7.8)$ & $22.0(8.7)$ \\
\hline Female, N(\%) & $41(46.1 \%)$ & $43(59.7 \%)$ & $20(52.6 \%)$ & $23(67.6 \%)$ \\
\hline ppFEV $_{1}$, Mean (SD) & $65.8(15.8)$ & $60.8(16.9)$ & $60.9(16.3)$ & $60.6(17.7)$ \\
\hline \multicolumn{5}{|l|}{ BMI, N(\%) } \\
\hline Normal Weight & 75 (84.3\%) & 57 (79.2\%) & $28(73.7 \%)$ & $29(85.3 \%)$ \\
\hline Obese & $3(3.4 \%)$ & $2(2.8 \%)$ & $2(5.3 \%)$ & $0(0 \%)$ \\
\hline Overweight & $9(10.1 \%)$ & $8(11.1 \%)$ & $6(15.8 \%)$ & $2(5.9 \%)$ \\
\hline Underweight & $2(2.3 \%)$ & $5(6.9 \%)$ & $2(5.3 \%)$ & $3(8.8 \%)$ \\
\hline Sweat chloride, Mean (SD) & $100.5(9.5)$ & $99.9(11.3)$ & $100(11.4)$ & $99.8(11.2)$ \\
\hline History of pancreatic insufficiency, N(\%) & $81(91.0 \%)$ & $68(94.4 \%)$ & 35 (92.1\%) & $33(97.1 \%)$ \\
\hline Use of inhaled cycling antibiotic, N(\%) & $37(41.6)$ & $19(26.4 \%)$ & $8(21.1 \%)$ & $11(32.4 \%)$ \\
\hline
\end{tabular}




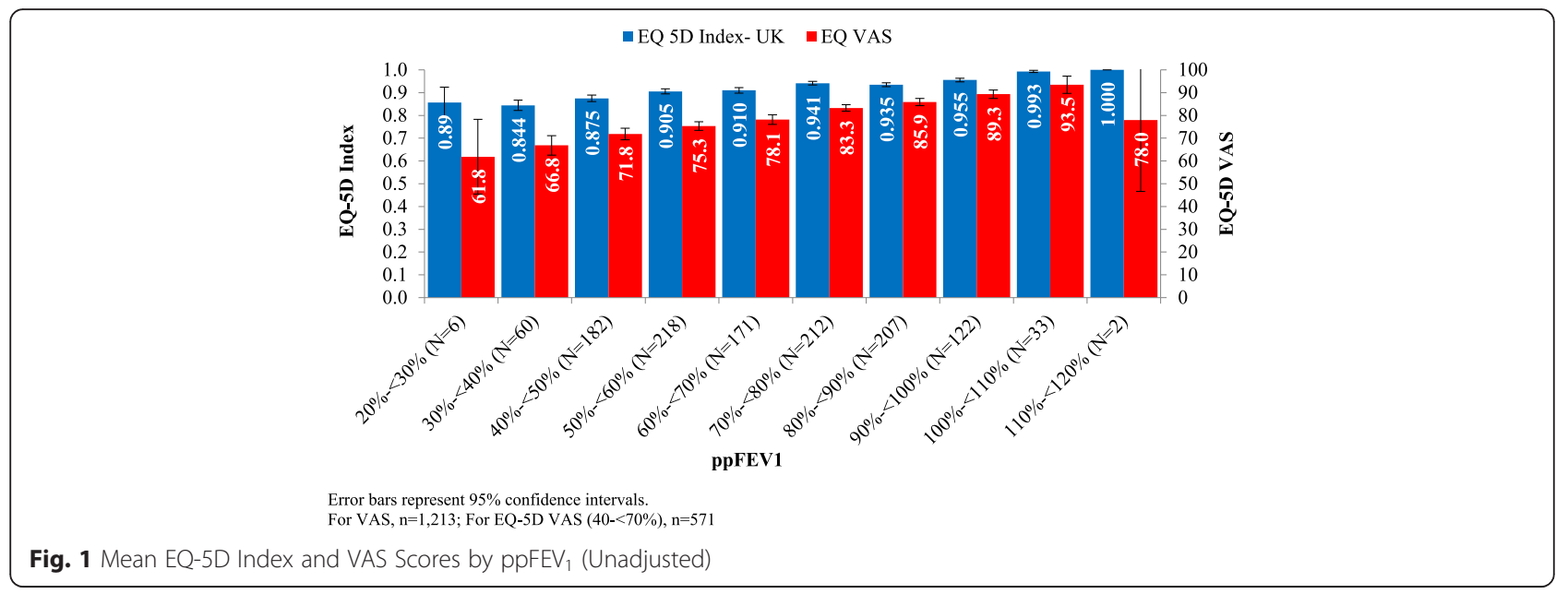

$28.6 \%$ for pain/discomfort, and $16.7 \%$ for both anxiety and depression and mobility. Problems were reported on at least one dimension of the EQ-5D for $52.4 \%$ of observations for hospitalized and non-hospitalized PEs and for $31.5 \%$ of observations that did not occur during a PE.

\section{Association of PEs, ppFEV 1 and EQ-5D measures}

In multivariate analyses, $\mathrm{PE}$ and low $\mathrm{ppFEV}_{1}$ values were significantly $(P<0.05)$ associated with a lower (worse) EQ-5D index (Table 3). When PEs were stratified by hospitalization status, experiencing a PE requiring hospitalization and low $\mathrm{pPEV}_{1}$ at the observation time were significant $(p<0.05)$ predictors of EQ-5D index. PEs not requiring hospitalization had a negligible positive effect $(P=0.965)$. In VAS models, PEs, PEs requiring hospitalization, and low $\mathrm{ppFEV}_{1}$ were associated with lower (worse) VAS scores (Table 3). Interestingly PEs not requiring hospitalization had a greater (negative) effect on VAS scores as compared to PEs requiring hospitalization. Conclusions did not change when applying US, Belgium, Netherlands, European algorithms reflecting countryspecific preference weights and the VAS transformed algorithm (Additional file 1).

\section{Discussion}

This analysis provides the first assessment of the impact of both lung function and PEs on a generic HRQL measure in patients with CF. In post-hoc analyses of data from a clinical study of patients with CF ( $\geq 12$ years of age and a G551D-CFTR mutation), PEs, primarily those requiring hospitalization, were associated with lower HRQL as measured by the EQ-5D index. Mean EQ-5D

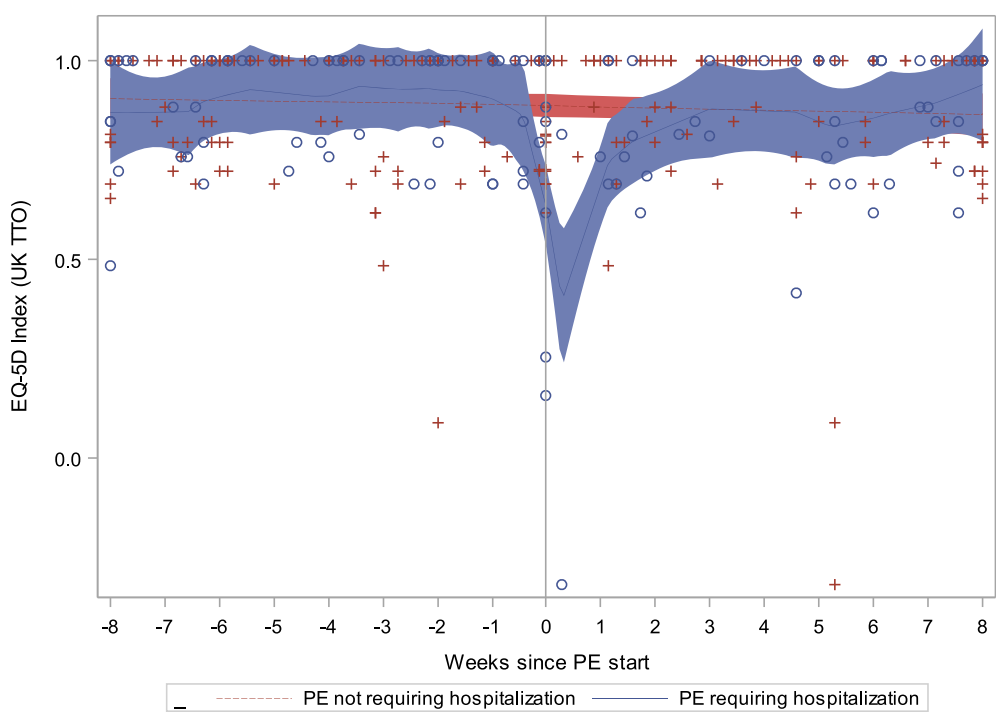

Fig. 2 EQ-5D Index by Time since PE Start and Hospitalization 


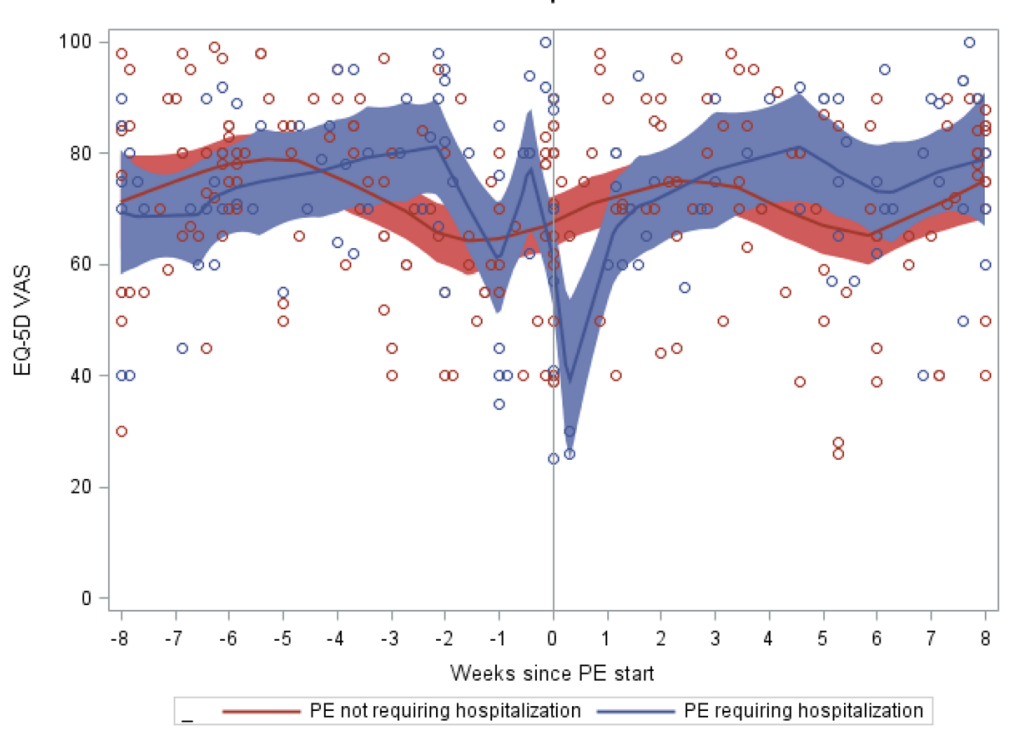

Fig. 3 EQ-5D VAS Scores by Time since PE Start and Hospitalization

index was lowest (worst) within one week (before or after PE start) for PEs requiring hospitalization. Similar findings were observed for ratings of health status as measured by VAS scores for all PEs regardless of whether they required hospitalization. Lung function as measured by $\mathrm{ppFEV}_{1}$ was also on average lowest (worst) within the one-week period before or after the study defined PE start date regardless of whether PEs required hospitalization or not. In multivariate analyses, experience of any PEs, PEs requiring hospitalization and low $\mathrm{ppFEV}_{1}$ were identified as independent negative predictors of EQ-5D index and VAS scores.

Our study findings are consistent with those reported by others $[5,6]$. A prior meta-analysis has identified PEs and ppFEV $_{1}$ as predictors of disease-specific HRQL and symptoms as measured by Cystic Fibrosis Questionnaire-

Table 2 EQ-5D index, VAS scores and ppFEV $_{1}$ by time since Pulmonary Exacerbation Start

\begin{tabular}{|c|c|c|c|c|c|c|c|}
\hline & \multirow[b]{2}{*}{$\mathrm{n}$} & \multicolumn{2}{|l|}{ EQ-5D Index } & \multicolumn{2}{|l|}{ EQ-5D VAS } & \multicolumn{2}{|l|}{$\mathrm{ppFEV}_{1}$} \\
\hline & & LS Mean (SE) & $p$-value & LS Mean (SE) & $p$-value & LS Mean (SE) & $p$-value \\
\hline \multicolumn{8}{|l|}{ PE Requiring Hospitalization } \\
\hline$>8$ weeks prior & 162 & $0.904(0.020)$ & $<0.001$ & $72.8(2.1)$ & 0.015 & $60.9(2.1)$ & 0.043 \\
\hline$>4-8$ weeks prior & 27 & $0.884(0.030)$ & 0.001 & $73.3(3.1)$ & 0.038 & $60.1(2.5)$ & 0.226 \\
\hline$>1-4$ weeks prior & 20 & $0.905(0.034)$ & $<0.001$ & $73.8(3.5)$ & 0.046 & $64.7(2.6)$ & 0.002 \\
\hline Within 1 week of start: reference group & 23 & $0.760(0.033)$ & - & $65.3(3.4)$ & - & $57.6(2.5)$ & - \\
\hline$>1-4$ weeks post & 15 & $0.841(0.039)$ & 0.084 & $74.5(4.0)$ & 0.053 & $65.5(2.8)$ & 0.002 \\
\hline$>4-8$ weeks post & 29 & $0.847(0.030)$ & 0.022 & $72.7(3.1)$ & 0.052 & $63.4(2.4)$ & 0.004 \\
\hline$>8$ weeks post & 121 & $0.856(0.021)$ & 0.002 & $72.0(2.2)$ & 0.034 & $62.7(2.2)$ & 0.003 \\
\hline \multicolumn{8}{|l|}{ PE Not Requiring Hospitalization } \\
\hline$>8$ weeks prior & $305^{\mathrm{a}}$ & $0.883(0.016)$ & 0.787 & $72.1(1.5)$ & 0.021 & $59.3(1.7)$ & 0.029 \\
\hline$>4-8$ weeks prior & 54 & $0.912(0.024)$ & 0.252 & $73.3(1.9)$ & 0.014 & $58.3(1.8)$ & 0.336 \\
\hline$>1-4$ weeks prior & 36 & $0.876(0.029)$ & 0.988 & $72.5(2.2)$ & 0.071 & $60.6(1.9)$ & 0.015 \\
\hline Within 1 week of start: reference group & 38 & $0.876(0.027)$ & - & $68.0(2.1)$ & - & $57.1(1.9)$ & - \\
\hline$>1-4$ weeks post & 33 & $0.916(0.029)$ & 0.276 & $70.6(2.2)$ & 0.295 & $57.6(1.9)$ & 0.753 \\
\hline$>4-8$ weeks post & 47 & $0.849(0.025)$ & 0.396 & $71.9(2.0)$ & 0.082 & $57.5(1.8)$ & 0.748 \\
\hline$>8$ weeks post & 210 & $0.857(0.017)$ & 0.470 & $70.2(1.5)$ & 0.233 & $58.7(1.7)$ & 0.115 \\
\hline
\end{tabular}

Abbreviations: $p p F E V_{1}$ percent predicted forced expiratory volume in $1 \mathrm{~s}, V A S$ visual analog scale score, $L S$ least-squares ${ }^{\mathrm{a}} \mathrm{N}=304$ observations for $\mathrm{ppFEV}_{1}$ 
Table 3 Association of ppFEV 1 and Pulmonary Exacerbations with EQ-5D Index and VAS

\begin{tabular}{|c|c|c|c|}
\hline Parameter & Model 1: ppFEV 1 Only & Model 2: $\mathrm{ppFEV}_{1}+$ Any PE & Model 3: ppFEV 1 + PE Type \\
\hline \multicolumn{4}{|l|}{ EQ-5D Index } \\
\hline Intercept & $0.670(0.068)^{* *}$ & $0.678(0.067)^{* *}$ & $0.686(0.067)^{* *}$ \\
\hline $\mathrm{ppFEV}_{1}$ & $0.580(0.193)^{* *}$ & $0.561(0.193)^{*}$ & $0.535(0.193)^{*}$ \\
\hline $\mathrm{ppFEV}_{1}$ squared & $-0.305(0.135)^{* *}$ & $-0.294(0.135)^{*}$ & $-0.274(0.135)^{*}$ \\
\hline Any PE & - & $-0.026(0.013)^{*}$ & - \\
\hline PE (Hospitalization) & - & - & $-0.070(0.020)^{*}$ \\
\hline PE (No Hospitalization) & - & - & $0.001(0.016)$ \\
\hline \multicolumn{4}{|l|}{ EQ-5D VAS } \\
\hline Intercept & $57.79(2.38)^{* *}$ & $58.46(2.36)^{* *}$ & $58.48(2.36)^{* *}$ \\
\hline $\mathrm{ppFEV}_{1}$ & $32.14(3.23)^{* *}$ & $31.52(3.21)^{* *}$ & $31.49(3.21)^{* *}$ \\
\hline Any PE & - & $-4.4(1.08)^{* *}$ & - \\
\hline PE (Hospitalization) & - & - & $-3.82(1.69)^{*}$ \\
\hline PE (No Hospitalization) & - & - & $-4.75(1.34)^{* *}$ \\
\hline
\end{tabular}

Abbreviations: $P E$ pulmonary exacerbation, $p p F E V_{1}$ percent predicted forced expiratory volume in 1 second, VAS visual analog scale ${ }^{*} p<0.05{ }^{* *} p<0.001$

Revised (CFQ-R) scores [5]. In cross-sectional analyses of data from pediatric and adult patients with CF treated in a US Midwestern CF center, PEs were reported to have a profound negative impact on physical and psychosocial HRQL using the generic Short-Form (SF)-36 and the Child Health Questionnaire; the impact of $\mathrm{ppFEV}_{1}$ was reported to be relatively small compared to the impact of PEs [8]. In another study conducted in five UK CF centers, adolescent patients with CF and chronic Pseudomona aeruginosa infection who experienced PEs that required hospitalization reported poorer HRQL using the EQ-5D index as well as worse VAS scores compared to those with chronic infection and milder PEs (i.e., not requiring hospitalization) [6].

In our analyses, ceiling effects were high, particularly for the EQ-5D index and in patients with no lung dysfunction or mild lung dysfunction as well as among those with less severe disease (i.e., patients who did not experience PEs). Mixed results have been reported by others regarding the association between physiologic measures of pulmonary disease and $\mathrm{CF}$, particularly $\mathrm{ppFEV}_{1}$, nutritional indices and HRQL [8, 25-27]. Patients with the most severe disease as measured by pulmonary function measures and weight for height have been reported to rate their CF as "above/well above average" compared to other patients with CF whereas physician's ratings of disease severity were positively correlated with clinical findings [28]. Yi et al.'s study of adolescents with CF that employed preference elicitation methods (e.g., time tradeoff, standard gamble) found that HRQL was poorly associated with lung function [7]. In our subgroup analysis, HRQL impacts of PEs requiring hospitalization were still apparent after exclusion of patients who did not experience a decline in absolute $\mathrm{ppFEV}_{1}$ of 10 percentage points or higher prior to the PE start date.
In summary, our results are supportive of prior suggestions that "the powerful association of HRQL with exacerbations, and the weaker association with $\mathrm{FEV}_{1}$ percent predicted may imply that for patients with $\mathrm{CF}$ and their families, HRQL may have less to do with how severe one's underlying disease is, and more to do with the disruptive effect of exacerbations." [8]. While this may be more strongly observed for disease specific measures, there is an important role for including and assessing the impact of disease on generic measures, which are frequently used for cross-condition comparisons. The STRIVE trial appropriately included the EQ-5D as a generic measure of HRQL and the Cystic Fibrosis Questionnaire- Revised which has been reported elsewhere [29].

It should be noted that the EQ-5D index at the time of study initiation was high $($ mean $\approx 0.93$ ) leaving little room for improvement with study treatments. There are multiple possible explanations for these high values. Among others, STRIVE study criteria excluded patients with a history of any illness or condition that, in the opinion of the investigator, could confound the results of the study or pose an additional risk in administering study drug to the patients, acute respiratory illness or PE within four weeks of baseline, those with colonization with selected microorganisms, and patients with any "non-CF-related" illness within 2 prior weeks. High scores have been also reported at the time of study initiation in the TIGER clinical trial (Health State Utilities Index $[\mathrm{HUI}]=0.90$, and 0.83 using a feeling thermometer similar to the VAS) [15]. High values may also be explained by patients' adaptation and coping mechanisms leading to acceptance of their chronic condition. From the physician's point of view, patients with $\mathrm{CF}$ and their close companions 
may underestimate the severity of their disease and overestimate self-care, and such perceptions often remain constant over time even if the patient's health is clinically deteriorating [28]. Denial of physical symptoms as a protective means for psychosocial adaptation and emotional resiliency are suggested strategies for living in the present used by patients with chronic disease which may limit consideration of the full impact of their condition when evaluating health on a given day [15, 30-33].

While both EQ-5D and VAS are generic measures, they provide complementary information; the use of the EQ-5D index alone (a generic HQRL measure) may limit characterization of disease burden and health gains in patients with CF. The EQ-5D index is however, a necessary generic HRQL ("health-state utility") metric that is typically required in cost-effectiveness evaluations of new therapies [34]. In our analysis, the EQ-5D VAS measure showed greater ability to discriminate disease severity (as measured by ppFEV 1 and PEs respectively) than the EQ-5D index. This broader range of response on the VAS measure, as compared to the EQ-5D index, which has also been reported by others [6], may point to health constructs or dimensions that may not be fully captured by the EQ-5D questionnaire. Some dimensions of the EQ$5 \mathrm{D}$, particularly self-care, are less likely to be impacted by CF; pain, discomfort, anxiety and depression have been reported to be most affected in previous studies $[16,35,36]$ but others may not be well represented by this generic HRQL measure. Use of disease-specific measures (e.g., CFQ-R) alongside generic instruments, per guidance, [1] should provide complementary assessment of patient-reported symptoms and HRQL.

The current analysis does have a number of limitations. EQ-5D measures were not assessed at the time of PE start as this was not part of the original study design. Sample size was small for ppFEV ${ }_{1}$ category $<40 \%$ and for some of the PE-related analytical windows employed in our analyses. Patients in clinical practice may differ from those participating in the STRIVE clinical trial and caution should be used in generalization of study findings. Also, the STRIVE clinical study included only patients with CF and the G551D-CFTR mutation. Finally, the EQ-5D was designed for use in populations 18 years of age or older, [19] whereas this study included adolescent patients as young as 12 years of age. However, it should be noted that some previous research has indicated that as long as the language and concepts used within the instrument are understood, the EQ-5D may be used in adolescents (1218 years) with adequate functioning ([37] as cited in [38]).

\section{Conclusion}

In summary, in a clinical study of patients with $\mathrm{CF}$ ( $\geq 12$ years of age and a G551D-CFTR mutation), PEs, primarily those requiring hospitalization, were associated with low EQ-5D index and VAS scores. The impact of $\mathrm{ppFEV}_{1}$ was relatively smaller. Reducing PEs, in particular those requiring hospitalization, is likely to improve HRQL among these patients.

\section{Availability of data and materials}

Supplemental data and materials are available upon request.

\section{Ethics approval and consent to participate}

This was a secondary analysis of the STRIVE clinical trial data. Please see original trial [18] for information on ethics approval.

\section{Additional file}

Additional file 1: Mixed model parameter estimates in models predicting EQ-5D utility values using US, European and Belgium EQ-5D algorithms and VAS-transformed utilities. (DOCX $15 \mathrm{~kb}$ )

\section{Abbreviations}

BMI: body mass index; CF: cystic fibrosis; $\mathrm{HRQL}$ : health-related quality of life; LOESS: non-parametric local regression smoothing; PE: pulmonary exacerbation; ppFEV : $_{1}$ percent predicted forced expiratory volume in 1 second; VAS: visual analog scale.

\section{Competing interests}

This study was sponsored by Vertex Pharmaceuticals Incorporated, Boston, Massachusetts. Brenda Castiglione is an employee of Vertex Pharmaceuticals and owns stock or stock options of Vertex Pharmaceuticals. Caitlyn Solem, and Marc Botteman are employees of Pharmerit International, a healthservices research consultancy that received fees for conducting analyses and manuscript preparation. Sizhu Liu was an employee of Pharmerit International and Montserrat Vera-Llonch was an employee of Vertex Pharmaceuticals at the time the study was undertaken.

\section{Authors' contributions}

C S, M V L; and M B contributed to study design and conceptualization. C S and $S \mathrm{~L}$ undertook data analysis. All authors contributed to data

interpretation, manuscript drafting, and approved the final manuscript.

\section{Acknowledgements}

We extend our deepest appreciation to the adolescents and adults with CF who participated in the STRIVE study. The authors are grateful for the analytical suggestions provided by Dr. Mark Higgins.

\section{Author details}

${ }^{1}$ Pharmerit International, 4350 East West Hwy, Suite 430, Bethesda, MD 20814, USA. Vertex Pharmaceuticals, 50 Northern Ave, Boston, MA 02210, USA.

Received: 20 October 2015 Accepted: 7 April 2016

Published online: 21 April 2016

\section{References}

1. Patrick DL, Deyo RA. Generic and disease-specific measures in assessing health status and quality of life. Med Care. 1989;27(3 Suppl):S217-32. Review. PubMed PMID: 2646490.

2. Sheppard MN, Nicholson AG. The pathology of cystic fibrosis. Curr Diagn Pathol. 2002:8(1):50-9.

3. Boucher RC. Airway surface dehydration in cystic fibrosis: pathogenesis and therapy. (0066-4219 (Print)).

4. de Boer K, Vandemheen KL, Tullis E, Doucette S, Fergusson D, Freitag A, Paterson N, Jackson M, Lougheed MD, Kumar V, Aaron SD. Exacerbation frequency and clinical outcomes in adult patients with cystic fibrosis. Thorax. 
2011;66(8):680-5. doi:10.1136/thx.2011.161117. Epub 2011 Jun 15. PubMed PMID: 21680566

5. Habib AR, Manji J, Wilcox PG, Javer AR, Buxton JA, Quon BS. A systematic review of factors associated with health-related quality of life in adolescents and adults with cystic fibrosis. Ann Am Thorac Soc. 2015;12(3):420-8. doi:10. 1513/AnnalsATS.201408-3930C. Review. PubMed PMID: 25642976.

6. Bradley JM, Blume SW, Balp MM, Honeybourne D, Elborn JS. Quality of life and healthcare utilisation in cystic fibrosis: a multicentre study. Eur Respir J. 2013;41(3):571-7.

7. Yi MS, Britto MT, Wilmott RW, Kotagal UR, Eckman MH, Nielson DW, et al. Health values of adolescents with cystic fibrosis. J Pediatr. 2003;142(2):133-40.

8. Britto MT, Kotagal UR, Hornung RW, Atherton HD, Tsevat J, Wilmott RW. Impact of recent pulmonary exacerbations on quality of life in patients with cystic fibrosis. Chest. 2002;121(1):64-72.

9. Taylor-Robinson D, Whitehead M, Diderichsen F, Olesen HV, Pressler T, Smyth RL, et al. Understanding the natural progression in \%FEV1 decline in patients with cystic fibrosis: a longitudinal study. Thorax. 2012;67(10):860-6.

10. Busschbach JJ, Horikx PE, van den Bosch JM, Brutel de la Riviere A, de Charro FT. Measuring the quality of life before and after bilateral lung transplantation in patients with cystic fibrosis. Chest. 1994;105(3):911-7.

11. Rowley PT, Loader S, Kaplan RM. Prenatal screening for cystic fibrosis carriers: an economic evaluation. Am J Hum Genet. 1998;63(4):1160-74.

12. Veenstra DL, Harris J, Gibson RL, Rosenfeld M, Burke W, Watts C. Pharmacogenomic testing to prevent aminoglycoside-induced hearing loss in cystic fibrosis patients: potential impact on clinical, patient, and economic outcomes. Genet Med. 2007;9(10):695-704.

13. Ramsey SD, Patrick DL, Albert RK, Larson EB, Wood DE, Raghu G. The costeffectiveness of lung transplantation. A pilot study. University of Washington Medical Center Lung Transplant Study Group. Chest. 1995;108(6):1594-601.

14. Santana MJ, Feeny D, Ghosh S, Lien DC. Patient-reported outcome 2 years after lung transplantation: does the underlying diagnosis matter? Patient Relat Outcome Meas. 2012;3:79-84.

15. Dewitt EM, Grussemeyer CA, Friedman JY, Dinan MA, Lin L, Schulman KA, et al. Resource use, costs, and utility estimates for patients with cystic fibrosis with mild impairment in lung function: analysis of data collected alongside a 48-week multicenter clinical trial. Value Health. 2012;15(2):277-83.

16. Connolly M, Johnson J, Brown NE, Montgomery M, Zuberbuhler P: Development Of A Disease-Specific Health-Related Quality Of Life Instrument For People With Cystic Fibrosis. In: Working Paper 00-11. Edited by http://citeseerx.ist.psu.edu/viewdoc/download; jsessionid=055FD26C437F1001EAC55AFAE214190D?doi=10.1.1.617 8566\&rep=rep1\&type=pdf: Institute of Health Economics, Alberta, Canada.

17. S Acaster, B Pinder, J Osmond, C Mukuria. Mapping the Cystic Fibrosis Questionnaire - Revised (CFQ-R) to a Preference Based Utility Index. 36th European Cystic Fibrosis Society Conference 2013, June 12-15, 2013(Lisbon, Portugal).

18. Ramsey BW, Davies J, McElvaney NG, Tullis E, Bell SC, Drevinek P, et al. A CFTR potentiator in patients with cystic fibrosis and the G551D mutation. N Engl J Med. 2011;365(18):1663-72.

19. About EQ-5D [http://www.euroqol.org/about-eq-5d.html]

20. Dolan P. Modeling valuations for EuroQol health states. Med Care. 1997;35(11):1095-108.

21. Lamers LM, McDonnell J, Stalmeier PF, Krabbe PF, Busschbach JJ. The Dutch tariff: results and arguments for an effective design for national EQ-5D valuation studies. Health Econ. 2006;15(10):1121-32.

22. Cleemput I. Economic evaluation in renal transplantation: outcome assessment and cost-utility of non-compliance. Leuven: Acco; 2003.

23. Greiner W, Weijnen T, Nieuwenhuizen M, Oppe S, Badia X, Busschbach J, et al. A single European currency for EQ-5D health states. Results from a sixcountry study. Eur J Health Econ. 2003;4(3):222-31.

24. Torrance GW. Social preferences for health states: an empirical evaluation of three measurement techniques. Socio Econ Plan Sci. 1976;10(3):129-36.

25. S Acaster BP, J Osmond, C Mukuria. Mapping the Cystic Fibrosis Questionnaire - Revised (CFQ-R) to a Preference Based Utility Index. 36th European Cystic Fibrosis Society Conference 2013. 2013;June 12-15, 2013(Lisbon, Portugal).

26. Czyzewski DI, Mariotto MJ, Bartholomew LK, LeCompte SH, Sockrider MM. Measurement of quality of well being in a child and adolescent cystic fibrosis population. Med Care. 1994;32(9):965-72. PubMed PMID: 8090048.
27. Powers PM, Gerstle R, Lapey A. Adolescents with cystic fibrosis: family reports of adolescent healthrelated quality of life and forced expiratory volume in one second. Pediatrics. 2001;107(5):E70. PubMed PMID: 11331720.

28. Abbott J, Dodd M, Webb AK. Different perceptions of disease severity and self care between patients with cystic fibrosis, their close companions, and physician. Thorax. 1995;50(7):794-6. PubMed PMID:7570419; PubMed Central PMCID: PMC474657.

29. Quittner A, Suthoff E, Rendas-Baum R, Bayliss MS, Sermet-Gaudelus I, Castiglione $B$, et al. Effect of ivacaftor treatment in patients with cystic fibrosis and the G551D-CFTR mutation: patient-reported outcomes in the STRIVE randomized, controlled trial. Health Qual Life Outcomes. 2015;13:93.

30. Jedlicka-Köhler I, Götz M. Interventional assessment of physical and mental health in children and adolescents with cystic fibrosis. Scand J Gastroenterol Suppl. 1988;143:34-7. PubMed PMID: 3164507.

31. Charmaz K. Good days, bad days: The self and chronic illness in time. Rutgers University Press; 1991

32. Moola F, Norman M. 'Down the rabbit hole': enhancing the transition process for youth with cystic fibrosis and congenital heart disease by re-imagining the future and time. Child Care Health Dev. 2011;37(6):841-51.

33. Jessup M, Parkinson C. "All at sea": The experience of living with cystic fibrosis. Qual Health Res. 2010;20(3):352-64.

34. Brazier J, Rowen D. NICE DSU technical support document 11: alternatives to EQ-5D for generating health state utility values. 2011.

35. Eidt-Koch D, Mittendorf T, Greiner W. Cross-sectional validity of the EQ-5D-Y as a generic health outcome instrument in children and adolescents with cystic fibrosis in Germany. BMC Pediatr. 2009;9:55.

36. Johnson JA, Connolly M, Zuberbuhler P, Brown NE. Health-related quality of life for adults with cystic fibrosis: a regression approach to assessing the impact of recombinant human DNase. Pharmacotherapy. 2000;20(10):1167-74.

37. Hennessy S, Kind P. Measuring health status in children: Developing and testing a child-friendly version of EQ-5D. In: 19th Plenary Meeting of the EuroQol Group: 2002; York, United Kingdom; 2002.

38. Wille N, Badia X, Bonsel G, Burstrom K, Cavrini G, Devlin N, et al. Development of the EQ-5D-Y: a child-friendly version of the EQ-5D. Qual Life Res. 2010;19(6):875-86.

\section{Submit your next manuscript to BioMed Central and we will help you at every step:}

- We accept pre-submission inquiries

- Our selector tool helps you to find the most relevant journal

- We provide round the clock customer support

- Convenient online submission

- Thorough peer review

- Inclusion in PubMed and all major indexing services

- Maximum visibility for your research

Submit your manuscript at www.biomedcentral.com/submit
C Biomed Central 